\title{
Stereoselektive Alkylierung mit sichtbarem Licht durch Kombination von heterogener Photokatalyse mit Organokatalyse**
}

\author{
Maria Cherevatskaya, Matthias Neumann, Stefan Füldner, Christoph Harlander, \\ Susanne Kümmel, Stephan Dankesreiter, Arno Pfitzner, Kirsten Zeitler und Burkhard König*
}

\section{Dr. Wolf-Dieter Haack zum 80. Geburtstag gewidmet}

Die Anwendung von Sensibilisatoren zur Nutzung sichtbaren Lichts ist seit langem bekannt. ${ }^{[1]}$ Die Vielzahl aktueller Veröffentlichungen ${ }^{[2]}$ illustriert eindrucksvoll die breiten Anwendungsmöglichkeiten sichtbaren Lichts für verschiedene Reaktionen, z.B. die Umsetzung von Alkoholen zu Alkylhalogeniden, ${ }^{[3]}[2+2]-,^{[4]}[3+2]^{-[5]}$ und [4+2]-Cycloadditionen $^{[6]}$ oder Kohlenstoff-Kohlenstoff- ${ }^{[7]}$ sowie KohlenstoffHeteroatom-Bindungsknüpfungen. ${ }^{[8]}$ Die kooperative Kombination von Organokatalyse mit Photo-Redoxkatalyse, unter Verwendung von Ruthenium- oder Iridiumkomplexen ${ }^{[9]}$ oder organischen Farbstoffen ${ }^{[9 \mathrm{~d}]}$ als Photokatalysatoren, ermöglicht auch enantioselektive Reaktionen. ${ }^{[10]}$ Obwohl der Einsatz anorganischer Halbleiter wie Titandioxid für die photokatalytische Zersetzung von organischen Abfällen weit verbreitet ist ${ }^{[11]}$ gibt es in der organischen Synthese nur wenige Beispiele für Bindungsknüpfungen durch Halbleiter-Photokatalyse. ${ }^{[12]}$ Neben den von Kisch ${ }^{[13]}$ untersuchten CdS-vermittelten Bindungsknüpfungen sind auch oxidative C-CKupplungsreaktionen in Gegenwart von Titandioxid bekannt. ${ }^{[14]}$ Allerdings verlaufen Bindungsknüpfungen an heterogenen Photokatalysatoren normalerweise ohne Kontrolle der Stereochemie und ergeben daher Isomerengemische. ${ }^{[15,16]}$

Wir zeigen hier, dass durch die geeignete Kombination von stereoselektiver Organokatalyse mit heterogener PhotoRedoxkatalyse mit sichtbarem Licht Kohlenstoff-Kohlenstoff-Bindungen stereoselektiv und in guter Ausbeute geknüpft werden können. Dieser Ansatz vereint die Vorteile der heterogenen Katalyse (stabile und leicht abzutrennende Katalysatoren) mit der Stereoselektivität der homogenen Organokatalyse. ${ }^{[17,18]}$

Als erste Testreaktion für die Verwendung anorganischer, heterogener Photokatalysatoren wählten wir zunächst die von MacMillan und Mitarbeitern ${ }^{[9]}$ entwickelte enantioselektive $\alpha$-Alkylierung von Aldehyden (Tabelle 1). Fünf Halbleiter wurden eingesetzt: Kommerziell erhältliches weißes $\mathrm{TiO}_{2}$

[*] M. Sc. M. Cherevatskaya, M. Sc. M. Neumann, Dr. S. Füldner, M. Sc. C. Harlander, Dipl.-Chem. S. Kümmel, M. Sc. S. Dankesreiter, Prof. Dr. A. Pfitzner, Priv.-Doz. Dr. K. Zeitler, Prof. Dr. B. König Fakultät für Chemie und Pharmazie, Universität Regensburg Universitätsstraße 31, 93040 Regensburg (Deutschland) E-Mail: burkhard.koenig@chemie.uni-regensburg.de

[***] Diese Arbeit wurde von der Deutschen Forschungsgemeinschaft (GRK 1626, Chemische Photokatalyse) finanziell unterstützt. S.K. dankt der Deutschen Bundesstiftung Umwelt für ein Promotionsstipendium.

D. Hintergrundinformationen zu diesem Beitrag sind im WWW unter http://dx.doi.org/10.1002/ange.201108721 zu finden.
Tabelle 1: Enantioselektive Alkylierung mit MacMillans chiralem sekundärem Amin und verschiedenen anorganischen Halbleitern als Photokatalysatoren.

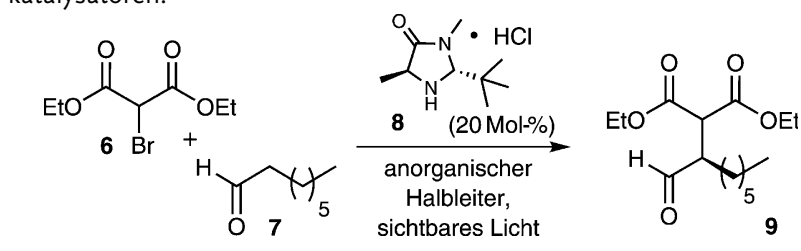

\begin{tabular}{|c|c|c|c|c|c|c|}
\hline Nr. & $\begin{array}{l}\text { Photo- } \\
\text { katalysator }^{[a]}\end{array}$ & $\begin{array}{l}\text { Wellenlänge }^{[\mathrm{b}]} \\
{[\mathrm{nm}]}\end{array}$ & $t[\mathrm{~h}]$ & $T\left[{ }^{\circ} \mathrm{C}\right]$ & $\begin{array}{c}\text { Ausb. } 9 \\
{[\%]^{[c]}}\end{array}$ & $\begin{array}{c}e e \\
{[\%]^{[d]}}\end{array}$ \\
\hline 1 & 1 & 440 & 20 & 20 & 55 & 71 \\
\hline 2 & $7^{[\mathrm{ee}]}$ & 440 & 20 & 20 & 60 & 72 \\
\hline 3 & $\mathbf{1}^{[f]}$ & 440 & 3 & 20 & 76 & 74 \\
\hline 4 & 1 & 440 & 20 & -10 & 40 & 83 \\
\hline 5 & 2 & 530 & 20 & 20 & 55 & 72 \\
\hline 6 & 2 & 530 & 20 & -10 & 65 & 81 \\
\hline 7 & 3 & 440 & 20 & 20 & 69 & 71 \\
\hline 8 & 3 & 440 & 20 & -10 & 40 & 84 \\
\hline 9 & 4 & 440 & 20 & 20 & 84 & 72 \\
\hline 10 & 4 & 440 & 20 & -10 & 49 & 83 \\
\hline 11 & $4^{[f]}$ & 455 & 3 & 20 & 41 & 71 \\
\hline 12 & $4^{[f]}$ & 455 & 10 & -10 & 69 & 80 \\
\hline
\end{tabular}

[a] $64 \mathrm{mg}$ Photokatalysator pro $1 \mathrm{mmol} 6$ in $2.5 \mathrm{~mL} \mathrm{CH} \mathrm{CN}$ (entgast). [b] Hochleistungs-LED $(440,455$ oder $530 \mathrm{~nm} \pm 10 \mathrm{~nm}, 3 \mathrm{~W}$, LUXEON, wie jeweils angegeben). [c] Ausbeute an isoliertem Produkt. [d] Bestimmt durch HPLC an chiraler Festphase oder NMR-Spektroskopie mithilfe eines chiralen Diols. ${ }^{[27]}[\mathrm{e}]$ Photokatalysator wiederverwendet. [f] Belichtung im Mikroreaktor in $1.5 \mathrm{~mL} \mathrm{CH}{ }_{3} \mathrm{CN}$.

(1) ${ }^{[19]}$ das gleiche Material, modifiziert durch kovalent an die Oberfläche gebundenen Phos-Texas-Red-Farbstoff zur Verbesserung der Absorption im sichtbaren Bereich (PhosTexas-Red- $\mathrm{TiO}_{2}, 2$ ), gelbes $\mathrm{PbBiO}_{2} \mathrm{Br}$, welches blaues Licht absorbiert, als Bulkphase (3) und in nanokristalliner Form (4). $\mathrm{TiO}_{2}$ (1) mit einer durchschnittlichen Partikelgröße von $21 \mathrm{~nm}$ ist ein stabiler und preiswerter Halbleiter mit einer Bandlücke von $3.2 \mathrm{eV}$; das nichtmodifizierte Pulver absorbiert jedoch aufgrund von Defekten und Oberflächenanlagerungen $^{[20]}$ nur schwach im Bereich bis $405 \mathrm{~nm}$. Der Absorptionsbereich kann aber durch Strukturmodifizierung ${ }^{[21]}$ oder durch Oberflächenmodifizierung mit einem Farbstoff $^{[22,23]}$ in den sichtbaren Bereich ausgedehnt werden. Der von Texas-Red abgeleitete Farbstoff 10 $^{[24]}$ (Schema 1) wurde kovalent an $\mathrm{TiO}_{2}$ gebunden, wodurch das bei $560 \mathrm{~nm}$ absorbierende Material 2 erhalten wurde (siehe die Hintergrundinformationen für die Synthese von $\mathbf{1 0}$ und die Charakterisierung von 2). $\mathrm{PbBiO}_{2} \mathrm{Br} \mathbf{3}$ und $\mathbf{4}$ wurden auf verschiedenen 


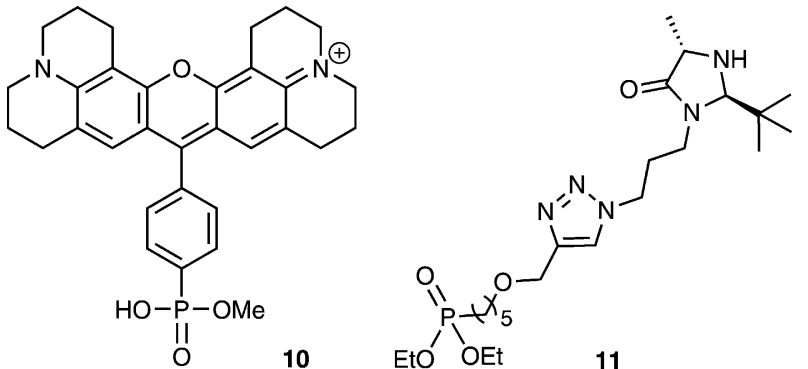

Schema 1. Substanzen für die kovalente Immobilisierung auf der $\mathrm{TiO}_{2}$ Oberfläche: Phos-Texas-Red (10) und der chirale Organokatalysator 11.

Synthesewegen hergestellt, was zu verschiedenen Partikelgrößen der Halbleiter führte: Bulkmaterial von $\mathrm{PbBiO}_{2} \mathrm{Br} 3$ mit einer Bandlücke von $2.47 \mathrm{eV}$ wurde durch Festphasensynthese bei hoher Temperatur hergestellt, ${ }^{[25]}$ während das nanokristalline Material 4 durch Synthese in wässriger Lösung erhalten wurde, wodurch sich eine durchschnittlich berechnete Partikelgröße von $(28 \pm 6) \mathrm{nm}$ und eine optische Bandlücke von $2.56 \mathrm{eV}$ ergaben. Gelbes CdS (5) hat eine Bandlücke von $2.4 \mathrm{eV}$ und wurde nach Literaturmethoden hergestellt. ${ }^{[6]}$

Die $\alpha$-Alkylierung von Aldehyd 7 in Gegenwart von 20 Mol-\% des sekundären Amins 8 als chiralem Katalysator und nichtmodifiziertem $\mathrm{TiO}_{2}$ liefert das gewünschte Produkt 9 in mäßiger Ausbeute, aber guter Enantioselektivität nach längerer Belichtungszeit (Tabelle 1, Nr. 1), da nur eine kleiner Bruchteil des sichtbaren Lichts bei $440 \mathrm{~nm}$ absorbiert wird. $\mathrm{TiO}_{2}$ kann wiederverwendet werden und zeigt dann ähnliche Ergebnisse (Nr. 2). Bei höheren Lichtintensitäten in einer Mikroreaktorapparatur (Nr. 3) verkürzt sich die Reaktionszeit auf $3 \mathrm{~h}$. Das Absenken der Reaktionstemperatur auf $-10^{\circ} \mathrm{C}$ erhöht die Enantioselektivität auf $83 \%$ ee, verlangsamt die Reaktion jedoch deutlich (Nr. 4). Mit dem oberflächenmodifizierten $\mathrm{TiO}_{2} 2$ kann die Reaktion auch mit grünem Licht (530 nm, Nr. 5 und 6) durchgeführt werden und liefert das Produkt in $65 \%$ Ausbeute und mit $81 \%$ ee bei $-10^{\circ} \mathrm{C}$. $\mathrm{PbBiO}_{2} \mathrm{Br}(3)$ absorbiert im sichtbaren Bereich und katalysiert die Reaktion bei Bestrahlung mit blauem Licht (Nr. 7 und 8). Allerdings ist dessen spezifische Oberfläche mit nur $0.17 \mathrm{~m}^{2} \mathrm{~g}^{-1}$ klein im Vergleich zu $\mathrm{TiO}_{2}\left(50 \mathrm{~m}^{2} \mathrm{~g}^{-1}\right)$. Das erklärt die noch immer eher lange Reaktionszeit. Nanokristallines $\mathrm{PbBiO}_{2} \mathrm{Br}(4)$ hat eine größere spezifische Oberfläche von $10.8 \mathrm{~m}^{2} \mathrm{~g}^{-1}$, und das Produkt kann bei Raumtemperatur und Belichtung bei $440 \mathrm{~nm}$ in $84 \%$ Ausbeute und mit $72 \%$ ee nach $20 \mathrm{~h}$ isoliert werden (Nr.9). Die Stereoselektivität konnte auch hier auf $83 \%$ ee bei $-10^{\circ} \mathrm{C}$ erhöht werden, aber mit geringerem Umsatz (Nr. 10). Der Mikroreaktor verkürzt die Reaktionszeit auf 3 bzw. 10 h, mit $69 \%$ Ausbeute und $80 \%$ ee (Nr. 11 und 12). Die Wiederverwendung von 4 ist möglich, allerdings führen schwarze organische Ablagerungen, die sich im Reaktionsverlauf abscheiden, zu einer deutlichen Verlangsamung der Umsetzung.

Der Mechanismus der Alkylierungsreaktion folgt vermutlich dem vorgeschlagenen Weg für die Photo-Redoxkatalyse (Schema siehe die Hintergrundinformationen): Einelektronentransfer vom Leitungsband des Halbleiters auf die halogenierte Carbonylverbindung liefert nach Eliminierung eines Bromidions ein $\alpha$-Carbonylradikal, welches an das durch Kondensation des chiralen Katalysators mit Octanal erhaltene Enamin addiert. Das $\alpha$-Aminoradikal wird dann durch ein Loch im Valenzband zum Iminiumion oxidiert, das schließlich Katalysator und Produkt freisetzt.

Um ein vollständig heterogenes Katalysatorsystem zu erhalten, synthetisierten wir den chiralen Amin-Phosphonatester 11 (Schema 1; Synthese siehe die Hintergrundinformationen) und immobilisierten die Substanz auf $\mathrm{TiO}_{2}$. Das so erhaltene Katalysatorsystem ist jedoch inaktiv, und unter identischen Reaktionsbedingungen wie zuvor wurde keine Produktbildung beobachtet. Die räumliche Nähe des Organokatalysators zur Oberfläche des Halbleiters führt möglicherweise zum schnellen oxidativen Photo-Abbau des sekundären Amins. Im Unterschied dazu wird der nichtimmobilisierte Katalysator, der in Lösung überwiegend als Enamin vorliegt, nur sehr selten als freies Amin in die Nähe der Halbleiteroberfläche kommen und ist so vor einem oxidativen Abbau geschützt.

Unsere Versuche, CdS (5) für diese Reaktion zu verwenden, waren nicht erfolgreich. Ein Vergleich der relevanten Potentiale des oft verwendeten Photokatalysators [Ru(bpy) $)_{3} \mathrm{Cl}_{2}$ und den untersuchten Halbleitern erklärt diese Beobachtung. $\left[\mathrm{Ru}(\mathrm{bpy})_{3}\right]^{+}$wird im Mechanismus als Elektronendonor mit einem Potential von $-1.33 \mathrm{~V}$ (gegen SCE) postuliert. Das Leitungsbandpotential von $\mathrm{TiO}_{2}$ bei $-2.0 \mathrm{~V}$ in Acetonitril ist ausreichend für diesen Schritt, während das entsprechende Potential für $\mathrm{CdS}$ in Acetonitril mit $-1.05 \mathrm{~V}$ zu niedrig erscheint (Abbildung 1). ${ }^{[28,29]}$ Das Potential zur Reduktion des angeregten $\left[\mathrm{Ru}(\mathrm{bpy})_{3}\right]^{2+*}$ durch Oxidation des $\alpha$-Aminoradikal-Intermediats wird hingegen auf $+0.84 \mathrm{~V}$

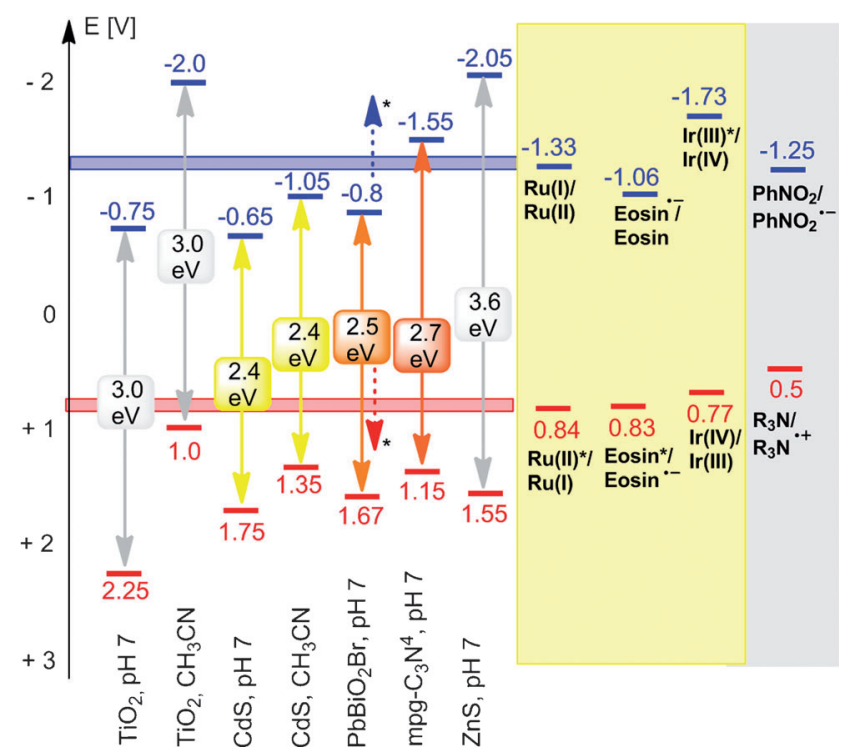

Abbildung 1. Bandlücken (in eV) von üblichen anorganischen Halbleitern im Vergleich zu Redoxpotentialen (in $\mathrm{V}$ gegen SCE) molekularer Photokatalysatoren und Redoxpotentialen einiger photokatalytischer Schlüsselschritte. *Geschätzte Änderung des $\mathrm{PbBiO}_{2} \mathrm{Br}$-Flachbandpotentials in Acetonitril. Angegebene Werte für Ru beziehen sich auf $\left[\mathrm{Ru}(\mathrm{bpy})_{3}\right]^{2+}$; Werte für Ir beziehen sich auf fac-[Ir(ppy $\left.)_{3}\right]$. bpy $=2,2^{\prime}$ Bipyridyl; ppy $=$ Phenylpyridyl. 
(gegen SCE) geschätzt, was zu den Loch-Potentialen von $\mathrm{TiO}_{2}(+1.0 \mathrm{~V})^{[29,30]}$ und $\mathrm{CdS}(+1.35 \mathrm{~V})$ passt (alle Werte gegen SCE in Acetonitril).

Die Kombination heterogener anorganischer mit homogenen organischen Katalysatoren ist auf Alkylierungen mit anderen Substraten wie Bromacetophenon (12a) übertragbar. Für die Umsetzung des schwieriger zu reduzierenden Dinitrobenzylbromids (12b) werden im Fall der homogenen Photokatalyse Iridiumkomplexe benötigt. ${ }^{\left[{ }^{\circ c}\right]}$ Die geschätzten Leitungsbandpotentiale von $\mathrm{TiO}_{2}$ und $\mathrm{PbBiO}_{2} \mathrm{Br}$ in Acetonitril (Abbildung 1) sollten jedoch ausreichen, und wir konnten tatsächlich die glatte Umsetzung zu den erwarteten Produkten in guter Ausbeute und mit hoher Stereoselektivität beobachten (Schema 2).
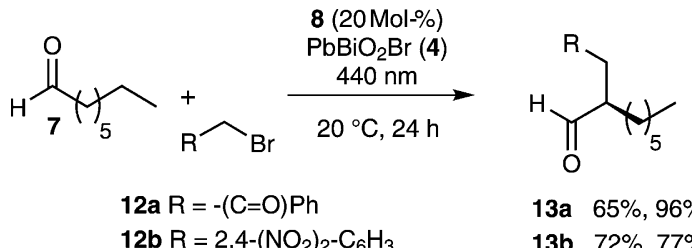

$12 b \mathrm{~b}=2,4-\left(\mathrm{NO}_{2}\right)_{2}-\mathrm{C}_{6} \mathrm{H}_{3}$

13a $65 \%, 96 \%$ ee

13b $72 \%, 77 \%$ ee

Schema 2. Alkylierung von 7 mit Bromacetophenon (12a) in $\mathrm{CH}_{3} \mathrm{CN}$ oder mit 2,4-Dinitrobenzylbromid (12b) in DMSO in Gegenwart von chiralem Amin 8 und $\mathrm{PbBiO}_{2} \mathrm{Br}$ (4) unter Verwendung von blauem Licht.

Kürzlich wurde über mehrere oxidative Kreuzkupplungen $^{[31]}$ an Tetrahydroisochinolinen durch homogene Photokatalyse mit Iridium- oder Rutheniumkomple$x n^{[5 b, c, 7 d, 8 b, d, 15,32]}$ oder organischen Farbstoffen ${ }^{[33]}$ wie Eosin $\mathrm{Y}^{[8 \mathrm{a}]}$ berichtet. Hierbei ist der photokatalytische Schlüsselschritt das reduktive Löschen des angeregten Chromophors, was zu einem Amin-Radikalkation führt, das anschließend in eine elektrophile Iminiumspezies umgewandelt werden kann. Möchte man anorganische Halbleiter für diese Reaktion nutzen, so ist nun das Potential der bei Bestrahlung erzeugten Löcher von Bedeutung. Basierend auf der Bandlücke und seinem Redoxpotential (siehe Abbildung 1), sollte CdS ein geeigneter heterogener Photokatalysator mit Absorption im sichtbaren Bereich sein, um durch Oxidation das gewünschte Amin-Radikalkation zu erzeugen. So ermöglicht die Kombination von Prolin als Organokatalysator mit $\mathrm{CdS}$ als anorganischem Photokatalysator die glatte Umsetzung von $\mathrm{N}$ Aryltetrahydroisochinolinen $\mathbf{1 4}$ in einer photooxidativen Mannich-Reaktion ${ }^{[32 b, 33 a]}$ mit den Ketonen $\mathbf{1 5}$ bei Bestrahlung mit blauem Licht $(\lambda=460 \mathrm{~nm})$. Die Produkte 16a-d, die sich aus der Reaktion mit acyclischen oder cyclischen Ketonen ergeben, werden in guten Ausbeuten von 76-89\% erhalten (Tabelle 2). ${ }^{[34]}$ Obwohl die Reaktion auch in $\mathrm{CH}_{3} \mathrm{CN}$ mit deutlich reduzierten Mengen an Keton erfolgreich abläuft (siehe Tabelle 2, Nr. 1a-c), ist die direkte Durchführung der Reaktion in reinem Keton speziell für preiswerte (flüssige) Ketone besonders einfach und praktikabel.

Die Flachbandpotentiale von einigen üblichen anorganischen und organischen Halbleitern sind in Abbildung $1 \mathrm{zu}-$ sammengefasst. ${ }^{[35]}$ Die Werte verschieben sich deutlich bei Änderung des $\mathrm{pH}$-Werts oder in verschiedenen organischen
Tabelle 2: Photokatalytische Mannich-Reaktion von N-Aryltetrahydroisochinolinen 14 mit Ketonen 15 und L-Prolin an CdS (5). ${ }^{[a]}$

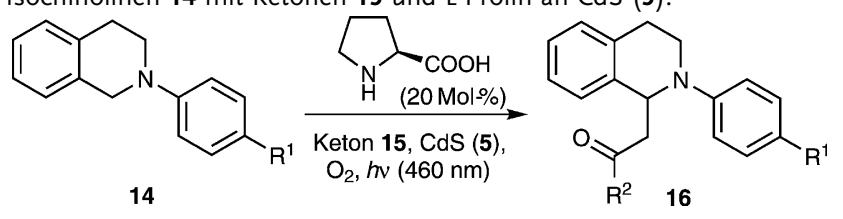

\begin{tabular}{|c|c|c|c|c|c|}
\hline Nr. & $\mathrm{R}^{1}$ & Keton & Produkt & $t[\mathrm{~h}]$ & Ausb. $[\%]^{[b]}$ \\
\hline $1 \mathrm{a}$ & & 0 & & 24 & $86^{[c]}$ \\
\hline $1 \mathrm{~b}$ & & & & 24 & $90^{[d]}$ \\
\hline $1 \mathrm{c}$ & $\mathrm{H}$ & 15 & & 24 & $100^{[\mathrm{e}]}$ \\
\hline $1 \mathrm{~d}$ & & & $16 a$ & 24 & 87 \\
\hline
\end{tabular}

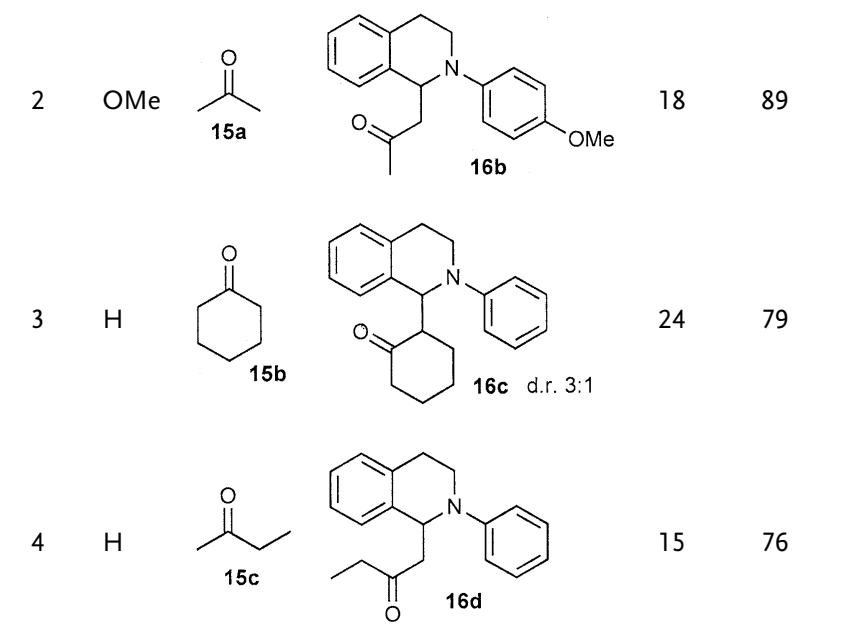

[a] Reaktionsbedingungen (soweit nicht anders vermerkt): Amin (1 Äquiv.) und L-Prolin (0.2 Äquiv.) in einer $5 \mathrm{mg} \mathrm{mL}^{-1}$-Mischung von $\mathrm{CdS}$ in reinem Keton durchgeführt $\left(c_{\mathrm{Amin}}=0.25 \mathrm{~mol} \mathrm{~L}^{-1}\right)$. Die Reaktionen wurden in Schlenk-Röhrchen mit einem Sauerstoffballon durchgeführt und mit Hochleistungs-LEDs (460 nm) für die angegebene Zeit belichtet. [b] Angegebene Ausbeuten entsprechen isoliertem Produkt. [c] Reaktion in $\mathrm{CH}_{3} \mathrm{CN}$ durchgeführt mit 2 Äquiv. Aceton; Umsatz bestimmt durch GC-Analyse. [d] Reaktion in $\mathrm{CH}_{3} \mathrm{CN}$ durchgeführt mit 5 Äquiv. Aceton; Umsatz bestimmt durch GC-Analyse. [e] Reaktion in $\mathrm{CH}_{3} \mathrm{CN}$ durchgeführt mit 10 Äquiv. Aceton; Umsatz bestimmt durch GC-Analyse.

Lösungsmitteln; die dafür zurzeit erhältlichen Daten sind sehr begrenzt. Dennoch erlaubt der Vergleich von HalbleiterFlachbandpotentialen mit den benötigten Potentialen für katalytische Schlüsselschritte von bekannten Photo-Redoxkatalysatoren (z.B. Ru-, Ir-Komplexe, Xanthenfarbstoffe usw.) die Vorhersage geeigneter Kombinationen von anorganischen Halbleitern mit Organokatalysatoren.

Wir konnten zeigen, dass die richtige Kombination von heterogener Halbleiter-Photokatalyse mit chiralen Organokatalysatoren stereoselektive Bindungsknüpfungen mithilfe von sichtbarem Licht ermöglicht. Die dabei erzielten Ausbeuten und Stereoselektivitäten sind vergleichbar mit zuvor berichteten Werten für homogenkatalysierte Reaktionen mit Übergangsmetallkomplexen oder organischen Farbstoffen. Passen die Redoxpotentiale der Substrate und die Bandlücken der Photokatalysatoren zusammen, findet der Elektronentransfer in den hier beschriebenen Reaktionen zwischen den chiralen Reaktionsintermediaten und der Halbleiter- 
oberfläche statt. Eine kovalente Immobilisierung des Organokatalysators auf der Halbleiteroberfläche ist allerdings nicht möglich, da sie zur oxidativen Zersetzung des Aminkatalysators führt.

Die gute Verfügbarkeit anorganischer Halbleiter mit verschiedenen Bandlücken und Redoxpotentialen, ihre einfache Abtrennung aus der Reaktionsmischung und ihre folglich leichte Wiederverwendbarkeit machen sie zu idealen Partnern für chirale Organokatalysatoren in der stereoselektiven Photokatalyse.

Eingegangen am 11. Dezember 2011, veränderte Fassung am 17. Januar 2012 Online veröffentlicht am 13. März 2012

Stichwörter: Alkylierungen · Halbleiter · Organokatalyse . Photokatalyse · Sichtbares Licht

[1] a) A. J. Bard, Science 1980, 207, 139-144; b) F. Teplý, Collect. Czech. Chem. Commun. 2011, 76, 859-917; c) G. Pandey, M. K. Ghorai, S. Hazra, Pure Appl. Chem. 1996, 68, 653.

[2] Aktuelle Übersichtsartikel: a) J. M. R. Narayanam, C. R. J. Stephenson, Chem. Soc. Rev. 2011, 40, 102-113; b) Lit. [1b]; c) T. P. Yoon, M. A. Ischay, J. Du, Nat. Chem. 2010, 2, 527-532; d) K. Zeitler, Angew. Chem. 2009, 121, 9969-9974; Angew. Chem. Int. Ed. 2009, 48, 9785-9789.

[3] C. Dai, J. M. R. Narayanam, C. R. J. Stephenson, Nat. Chem. 2011, 3, 140-145.

[4] a) M. A. Ischay, M. E. Anzovino, J. Du, T. P. Yoon, J. Am. Chem. Soc. 2008, 130, $12886-12887$; b) J. Du, T. P. Yoon, J. Am. Chem. Soc. 2009, 131, 14604-14605; c) M. A. Ischay, Z. Lu, T. P. Yoon, J. Am. Chem. Soc. 2010, 132, 8572-8574; d) J. Du, L. Ruiz Espelt, I. A. Guzei, T. P. Yoon, Chem. Sci. 2011, 2, 2115-2119.

[5] a) Z. Lu, M. Shen, T. P. Yoon, J. Am. Chem. Soc. 2011, 133, $1162-1164$; b) Y.-Q. Zou, L.-Q. Lu, L. Fu, N.-J. Chang, J. Rong, J.-R. Chen, W.-J. Xiao, Angew. Chem. 2011, 123, 7309-7313; Angew. Chem. Int. Ed. 2011, 50, 7171-7175; c) M. Rueping, D. Leonori, T. Poisson, Chem. Commun. 2011, 47, 9615-9617; d) S. Maity, M. Zhu, R. S. Shinabery, N. Zheng, Angew. Chem. 2012 124, 226-230; Angew. Chem. Int. Ed. 2012, 51, 222-226.

[6] a) A. E. Hurtley, M. A. Cismesia, Mi. A. Ischay, T. P. Yoon, Tetrahedron 2011, 67, 4442-4448; b) S. Lin, M. A. Ischay, C. G. Fry, T. P. Yoon, J. Am. Chem. Soc. 2011, 133, 19350-19353.

[7] Ausgewählte Beispiele aus der aktuellen Literatur: a) L. Furst, B. S. Matsuura, J. M. R. Narayanam, J. W. Tucker, C. R. J. Stephenson, Org. Lett. 2010, 12, 3104-3107; b) P. V. Pham, D. A Nagib, D. W. C. MacMillan, Angew. Chem. 2011, 123, 6243 6246; Angew. Chem. Int. Ed. 2011, 50, 6119-6122; c) J. D. Nguyen, J. W. Tucker, M. D. Konieczynska, C. R. J. Stephenson, J. Am. Chem. Soc. 2011, 133, 4160-4163; d) M. Rueping, S. Zhu, R. M. Koenigs, Chem. Commun. 2011, 47, 12709-12711; e) A. McNally, C. K. Prier, D. W. C. MacMillan, Science 2011, 334, 114-117; f) D. Kalyani, K. B. McMurtrey, S. R. Neufeldt, M. S. Sanford, J. Am. Chem. Soc. 2011, 133, 18566; g) D. A. Nagib, D. W. C. MacMillan, Nature 2011, 480, 224-228.

[8] C-P-Bindungsknüpfungen: a) D. P. Hari, B. König, Org. Lett. 2011, 13, 3852-3855; b) M. Rueping, S. Zhu, R. M. Koenigs, Chem. Commun. 2011, 47, 8679-8681. Bildung von C-S-Bindungen: Y. Cheng, J. Yang, Y. Qu, P. Li, Org. Lett. 2011, 14, 98 101. C-N-Bindungsknüpfungen: J. Xuan, Y. Cheng, J. An, L.-Q. Lu, X.-X. Zhang, W.-J. Xiao, Chem. Commun. 2011, 47, 8337 8339.

[9] a) D. A. Nicewicz, D. W. C. MacMillan, Science 2008, 322, 77 80; b) D. A. Nagib, M. E. Scott, D. W. C. MacMillan, J. Am.
Chem. Soc. 2009, 131, 10875-10877; c) H.-W. Shih, M. N. Vander Wal, R. L. Grange, D. W. C. MacMillan, J. Am. Chem. Soc. 2010, 132, 13600 - 13603; d) M. Neumann, S. Füldner, B. König, K. Zeitler, Angew. Chem. 2011, 123, 981-985; Angew. Chem. Int. Ed. 2011, 50, 951-954.

[10] Grundlegende und neuere Beispiele für enantioselektive photokatalytische Reaktionen mit UV-Licht: a) A. Bauer, F. Westkämper, S. Grimme, T. Bach, Nature 2005, 436, 1139-1140; b) C. Müller, A. Bauer, M. M. Maturi, M. C. Cuquerella, M. A. Miranda, T. Bach, J. Am. Chem. Soc. 2011, 133, 16689-16697. Übersichtsartikel zur enantioselektiven Photokatalyse mit Wasserstoffbrückentemplaten: c) C. Müller, T. Bach, Aust. J. Chem. 2008, 61, 557-564.

[11] a) A. Atyaoui, L. Bousselmi, H. Cachet, P. Pu, E. M. M. Sutter, J. Photochem. Photobiol. A 2011, 224, 71-79; b) F. Spadavecchia, G. Cappelletti, S. Ardizzone, C. L. Bianchi, S. Cappelli, C. Oliva, P. Scardi, M. Leoni, P. Fermo, Appl. Catal. B 2010, 96, 314-322; c) M. K. Seery, R. George, P. Floris, S. C. Pillai, J. Photochem. Photobiol. A 2007, 189, 258-263; d) J. Tang, J. Ye, Angew. Chem. 2004, 116, 4563-4566; Angew. Chem. Int. Ed. 2004, 43, $4463-$ 4466.

[12] Ein aktives Forschungsgebiet ist der Gebrauch von anorganischen Halbleitern als Photokatalysatoren zur Wasserspaltung und Wasserstoff-Erzeugung. Aktuelle Übersichtsartikel: a) X. Chen, S. S. Mao, Chem. Rev. 2007, 107, 2891-2959; b) R. M. Navarro Yerga, M. C. Álvarez-Galván, F. del Valle, J. A. Villoria de La Mano, J. L. G. Fierro, ChemSusChem 2009, 2, 471485; c) H. Xu, R. Q. Zhang, A. N. C. Ng, A. B. Djurišić, H. T. Chan, W. K. Chan, S. Y. Tong, J. Phys. Chem. C 2011, 115, 19710-19715; d) M. Antoniadou, V. M. Daskalaki, N. Balis, D. I. Kondarides, C. Kordulis, P. Lianos, Appl. Catal. B 2011, 107, $188-196$.

[13] a) H. Kisch, W. Schindler, J. Photochem. Photobiol. A 1993, 103, 257-264; b) H. Kisch, W. Linder, Chem. Unserer Zeit 2001, 35, 250-257; c) M. Gärtner, H. Kisch, Photochem. Photobiol. Sci. 2007, 6, 159-164; d) N. Zeug, J. Bücheler, H. Kisch, J. Am. Chem. Soc. 1985, 107, 1459-1465; e) W. Hetterich, H. Kisch, Chem. Ber. 1987, 121, 15-20.

[14] L. Cermenati, C. Richter, A. Albini, Chem. Commun. 1998, $805-$ 806.

[15] Ausgewählte Beispiele mit Metall-organischen Gerüsten und Polymeren: a) Z. Xie, C. Wang, K. E. deKrafft, W. Lin, J. Am. Chem. Soc. 2011, 133, 2056-2059; b) C. Wang, Z. Xie, K. E. deKrafft, W. Lin, J. Am. Chem. Soc. 2011, 133, 13445-13454.

[16] Ausgewählte Beispiele unter Verwendung von organischen Halbleitern: a) F. Su, S. C. Mathew, L. Möhlmann, M. Antonietti, X. Wang, S. Blechert, Angew. Chem. 2011, 123, 683-686; Angew. Chem. Int. Ed. 2011, 50,657-660; b) F. Su, S. C. Mathew, G. Lipner, X. Fu, M. Antonietti, S. Blechert, X. Wang, J. Am. Chem. Soc. 2010, 132, 16299-16301.

[17] Ausgewählte Übersichtsartikel zur Organokatalyse aus der aktuellen Literatur: a) S. Bertelsen, K. A. Jørgensen, Chem. Soc. Rev. 2009, 38, 2178-2189; b) B. List, Top. Curr. Chem. 2010, 291, 1-456 (Asymmetrische Organokatalyse); c) Chem. Rev. 2007, 107, 5413-5883 (Themenheft über Organokatalyse).

[18] Ein Beispiel aus der aktuellen Literatur zur Kombination von Organokatalyse und UV-Licht-TiO ${ }_{2}$-Photokatalyse: X.-H. Ho, M.-J. Kang, S.-J. Kim, E. D. Park, H.-Y. Jang, Catal. Sci. Technol. 2011, 1,923-926.

[19] P25 Degussa mit einem Anatas/Rutil-Verhältnis von 80:20 wurde verwendet.

[20] a) P. Roy, S. Berger, P. Schmuki, Angew. Chem. 2011, 123, $2956-$ 2995; Angew. Chem. Int. Ed. 2011, 50, 2904-2939; b) S. Higashimoto, N. Kitao, N. Yoshida, T. Sakura, M. Azuma, H. Ohue, Y. Sakata, J. Catal. 2009, 266, 279-285. c) Die Absorption des sichtbaren Lichts wurde Ablagerungen von organischem Material auf der Oberfläche von $\mathrm{TiO}_{2}$ zugeschrieben. 


\section{Angewandte}

[21] a) S. U. M. Khan, M. Al-Shahry, W. B. Ingler, Jr., Science 2002, 297, 2243-2245; b) X. Chen, L. Liu, P. Y. Yu, S. S. Maol, Science 2011, 331, 746-750.

[22] S. Füldner, R. Mild, H. I. Siegmund, J. A. Schroeder, M. Gruber, B. König, Green Chem. 2010, 12, 400-406.

[23] L. Xiong, F. Yang, L. Yan, N. Yan, X. Yang, M. Qiu, Y. Yu, J. Phys. Chem. Solids 2011, 72, 1104-1109.

[24] D. Chatterjee, V. R. Patnama, A. Sikdar, P. Joshi, R. Misra, N. N. Rao, J. Hazard. Mater. 2008, 156, 435 - 441 .

[25] A. Pfitzner, P. Pohla, Z. Anorg. Allg. Chem. 2009, 635, $1157-$ 1159 .

[26] M. Hopfner, H. Weiss, D. Meissner, F. W. Heinemann, H. Kisch, Photochem. Photobiol. Sci. 2002, 1, 696-703.

[27] Durchführung nach Lit. [9a]; Details siehe die Hintergrundinformationen.

[28] H. Kisch, Adv. Photochem. 2001, 26, $93-143$.

[29] Das Flachbandpotential von $\mathrm{PbBiO}_{2} \mathrm{Br}$ in Acetonitril wurde nicht bestimmt. Die erwartete Verschiebung des Potentials liegt zwischen +0.5 und $+1.0 \mathrm{~V}$ in Acetonitril verglichen mit Wasser, pH 7. Das wäre ausreichend für die beobachteten Reaktionen. Die Potentiale von $\mathrm{PbBiO}_{2} \mathrm{Br}$ in Wasser wurden bestimmt: $1.67 \mathrm{~V}$ für das Valenzband und $-0.80 \mathrm{~V}$ für das Leitungsband.

[30] Redoxpotential von Texas-Red: M. Torimura, S. Kurata, K. Yamada, T. Yokumaku, Y. Kamagata, T. Kanagawa, R. Kurane, Anal. Sci. 2001, 17, 155-160.
[31] Übersichtsartikel aus der aktuellen Literatur zur oxidativen Kupplung unter Verwendung von Oxidationsreagentien: a) C.-J. Li, Acc. Chem. Res. 2009, 42, 335-344; b) C. J. Scheuermann, Chem. Asian J. 2010, 5, 436-451; c) C. S. Yeung, V. M. Dong, Chem. Rev. 2011, 111, 1215-1292.

[32] a) A. G. Condie, J.-C. González-Gómez, C. R. J. Stephenson, J. Am. Chem. Soc. 2010, 132, 1464; b) M. Rueping, C. Vila, R. M. Koenigs, K. Poscharny, D. C. Fabry, Chem. Commun. 2011, 47, $2360-2362$

[33] a) Y. Pan, C. W. Kee, L. Chen, C.-H. Tan, Green Chem. 2011, 13, 2682-2685; b) Y. Pan, S. Wang, C. W. Kee, E. Dubuisson, Y. Yang, K. P. Loh, C.-H. Tan, Green Chem. 2011, 13, 3341-3344.

[34] Klussmann et al. berichteten über eine langsame Racemisierung der Produkte unter ihren oxidativen Reaktionsbedingungen: A. Sud, D. Sureshkumarz, M. Klussmann, Chem. Commun. 2009, 3169-3171. Ähnlich wie zuvor von Rueping (siehe Lit. [32b]) und Tan (siehe Lit. [33a]) beschrieben, zeigt unsere stereochemische Analyse auch nur geringe $e e$-Werte.

[35] Aktuelle Veröffentlichung zur Berechnung von Flachbandpotentialen von Halbleiteroxiden: M. C. Toroker, D. K. Kanan, N. Alidoust, L. Y. Isseroff, P. Liaob, E. A. Carter, Phys. Chem. Chem. Phys. 2011, 13, 16644-16654. 